\title{
3D reconstruction of human body model based on incremental Motion recovery structure
}

\author{
Shiheng Zhang, Shaopeng Zhang, Jianyang Chen, and Xiuling Wang* \\ College of Information Engineering, Inner Mongolia University of Technology, Hohhot, China
}

\begin{abstract}
D reconstruction of human body model is a very important research topic in $3 \mathrm{D}$ reconstruction and also a challenging research direction in engineering field. In this paper, the whole pipeline flow of 3D reconstruction of human body model based on incremental motion recovery structure is proposed. Use mobile phone to collect images from different angles and screen them; Secondly, feature extraction and matching under SIFT operator, sparse reconstruction of incremental motion recovery structure, dense reconstruction based on depth map and other processes are carried out. Poisson surface reconstruction is finally carried out to achieve model reconstruction. Experiments show that the effect subject of the reconstructed model is clear.
\end{abstract}

Keywords: 3D reconstruction, Images, Pipline flow.

\section{Introduction}

3D reconstruction of human models has been widely used in medicine ${ }^{[1]}$, film, animation, 3D games, online shopping and anthropometry. At present, there have been a lot of related scientific and technological researchers to discuss and study the 3D reconstruction of human model. Laser 3D scanning method mainly uses triangulation ${ }^{[2]}$, which has high measurement accuracy but high cost. Shi Le ${ }^{[3]}$ studied and generated automatic measurement system and personalized human body model by combining gauss skin color model and HSV color model. Wang $\mathrm{Li}^{[4]}$ studied and implemented a anthropometry and modeling method based on front side photos. Based on human contour information and Maya 3D software, a corresponding 3D human body model was established. However, the generated model is not personalized and authentic. Zhang Guangpian et al. ${ }^{[5]}$ proposed a new modeling method based on two-dimensional point cloud image to 3D human model. Xu Lei ${ }^{[6]}$ designed a hardware system for sequential image acquisition, which can llect multi-angle image sequences with high quality and high overlap. Then three - dimensional reconstruction is performed using sequence images.

However, the above reconstruction method has the disadvantage of high cost or insufficient personalization. Based on this, this paper proposes the overall pipeline process of $3 \mathrm{D}$ reconstruction of human model based on a series of $2 \mathrm{D}$ images. The main work is as follows: (1)Data set production: the equipment is used to collect the required pictures. The

\footnotetext{
* Corresponding author: wxltt3756@imut.edu.cn
} 
experimental data set of this paper contains 50 pictures. (2) The main pipeline process of reconstruction (camera calibration, feature extraction and matching, sparse reconstruction); (3) The pipeline process of subsequent reconstruction and improvement (dense reconstruction, surface reconstruction).Implement low-cost reconstruction process and personalize reconstruction model.

\section{Production of data set}

This chapter will elaborate on the process of making data sets. The data set is the basis of the whole reconstruction process, and the quality of the image plays a decisive role in the final model generation.

\subsection{Image acquisition equipment}

The images can be captured with relatively simple equipment, such as a smart phone or a professional camera. In this paper, 50 human body pictures were collected with smart phone. The main information of the collection device is listed in Table 1, including four related parameters.

Table 1. Main information of shooting equipment.

\begin{tabular}{cccc}
\hline Aperture & Exposure time & Speed of ISO & The focal length \\
\hline $\mathrm{f} / 1.8$ & $1 / 50 \mathrm{~s}$ & ISO-134 & $4 \mathrm{~mm}$ \\
\hline
\end{tabular}

\subsection{Image acquisition process}

In order to minimize the interference to the subject reconstruction object, it is preferred to collect images in a relatively empty environment. In this paper, the image acquisition environment is a football field. In addition, the influence of uneven lighting on image acquisition was considered to avoid weak texture and similar non-lambert surfaces ${ }^{[7]}$, so the collection work was carried out in the evening and the clothing texture was clear. Ensure that all the information of the reconstructed object is included in the data set, and take pictures at intervals around the object in order. There should be some information overlap (same feature point) between two adjacent pictures. First of all, the photographer was photographed at a height higher than that of the person being photographed, and the image information of the upper and middle parts of the human body was collected. Second, the middle and lower parts of the human body were photographed at knee level. Finally, according to the actual situation, the missing part is compensated to obtain the complete image information of the human model. Figure 1 is the data set of this paper.

\section{Initial reconstruction}

\subsection{Camera calibration}

Camera calibration is mainly to obtain the camera parameters and distortion correction, camera parameters and subsequent matching points for $3 \mathrm{~d}$ reconstruction of the image. There are two main methods of camera calibration: camera self-calibration and calibration based on calibrators ${ }^{[8]}$. Based on calibration (checkerboard chart), this paper takes 
checkerboard chart from multiple angles. Qualified checkerboard diagram (17) as shown in Figure 2:

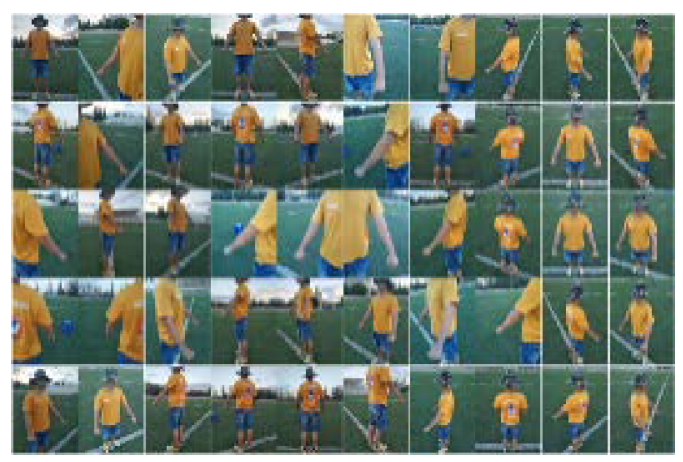

Fig. 1. Data set.

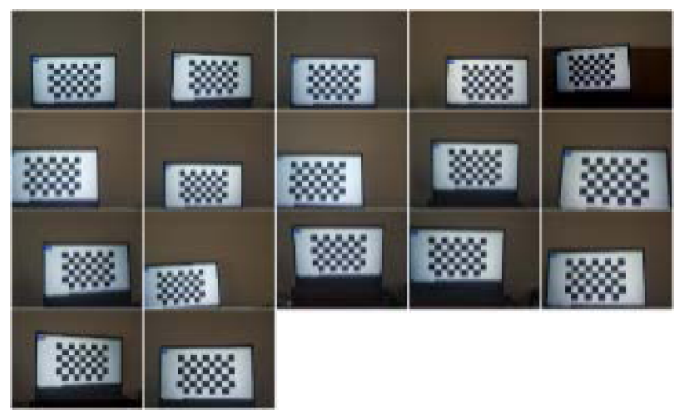

Fig. 2. Calibration pictures.

The internal parameter matrix of the camera obtained in Matlab is:

$$
\left[\begin{array}{ccc}
3178.661 & 0 & 0 \\
-0.347 & 3175.195 & 0 \\
2039.484 & 1509.924 & 1
\end{array}\right]
$$

The distortion matrix is:

$$
\left[\begin{array}{lllll}
0.3452 & -1.6527 & -0.0001 & -0.0003 & 2.4296
\end{array}\right]
$$

Radial distortion correction:

$$
\begin{gathered}
x_{\text {distorted }}=x\left(1+k_{1} * r^{2}+k_{2} * r^{4}+k_{3} * r^{6}\right) \\
y_{\text {distorted }}=y\left(1+k_{1} * r^{2}+k_{2} * r^{4}+k_{3} * r^{6}\right)
\end{gathered}
$$

Tangential distortion correction:

$$
x_{\text {distorted }}=x+\left[2 * p_{1} * x * y+p_{2} *\left(r^{2}+2 * x^{2}\right)\right]
$$

According to the formula, the picture can be corrected. Before and after the correction, see Figure 3 and Figure 4: 


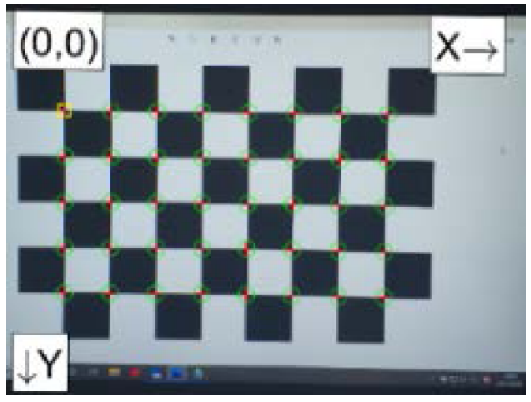

Fig. 3. Before correction.

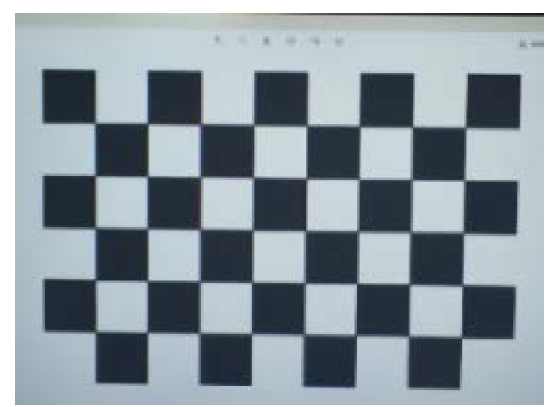

Fig. 4. After correction.

\subsection{Feature extraction and matching}

SIFT feature point detection has the advantages of high precision and relatively high execution efficiency. Sift is selected to extract feature points and perform feature matching ${ }^{[9]}$. Feature extraction and matching are shown in figures 5 and 6 :

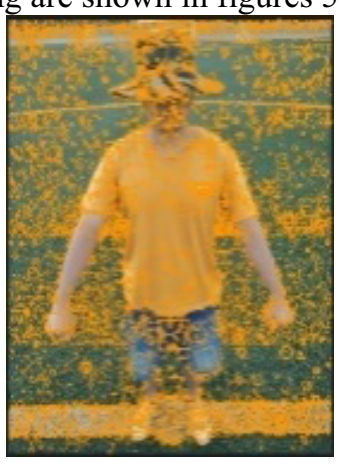

Fig. 5. Extraction.

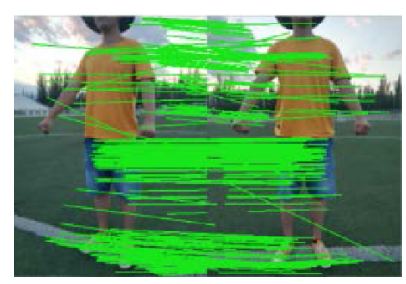

Fig. 6. Matching. 


\subsection{Sparse reconstruction}

This paper takes the SFM (structure from motion)as the core for sparse reconstruction ${ }^{[10]}$. The common motion recovery structures include incremental motion recovery structure, global motion recovery structure and hierarchical motion recovery structure. Among them, the incremental motion recovery structure has the advantages of strong robustness and high reconstruction accuracy. Therefore, the incremental motion recovery structure algorithm is adopted. The algorithm flow is shown in Figure 7:

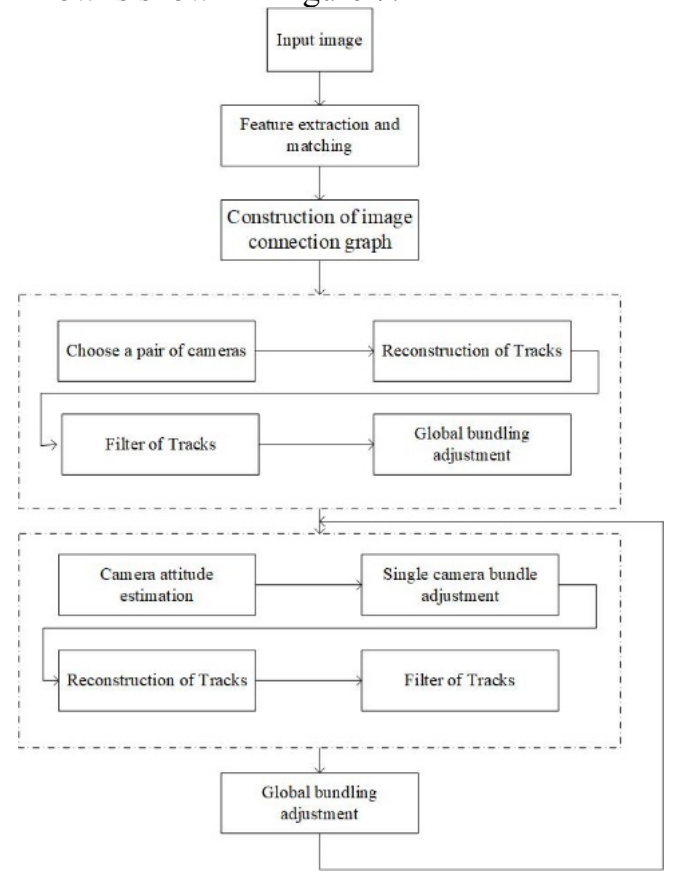

Fig. 7. Incremental SFM algorithm flow.

Incremental sparse reconstruction extracts features from a group of images and matches them, constructs the image connection graph, and optimizes the camera attitude and scene structure by binding adjustment to build a sparse model. Feature extraction and matching are carried out by SIFT algorithm, and then the images are connected to form a connection graph. Initialization is the combination of two images and trajectory reconstruction, filtering and binding. On this basis, camera attitude estimation, single camera bundling adjustment, trajectory reconstruction and filtering, and then reconstruct a new visual angle. Make global binding adjustment every few pictures, and finally get front (a), side (b) and back (c), as shown in Figure 8:

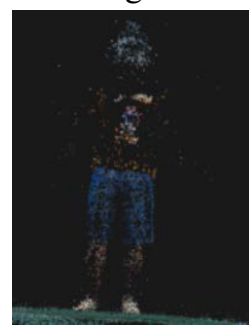

(a)

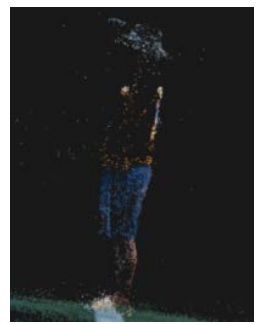

(b)

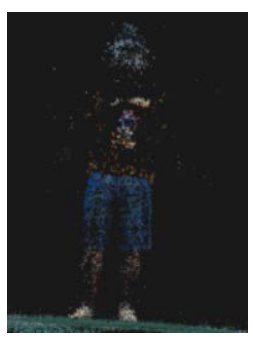

(c)

Fig. 8. Sparse clouds. 


\section{Follow-up reconstruction and improvement}

\subsection{Dense reconstruction}

After the sparse human point cloud is generated by incremental motion restoration structure algorithm, the sparse point cloud needs to be densely reconstructed to generate dense point cloud. This paper adopts the method of depth map fusion, constructs seeds from the reconstructed sparse points, constructs images with different resolutions, selects the global perspective, optimizes the spatial seed, and finally fuses the depth maps from different perspectives. The obtained depth map is shown in Figure 9:

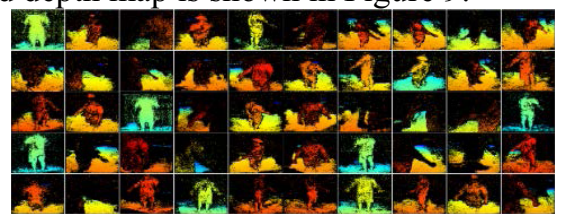

Fig. 9. Depth map.

Dense point clouds can be obtained after the fusion of the depth maps from different perspectives above. The dense point clouds obtained include front side (a), side side (b) and back side (c), as shown in Figure 10:

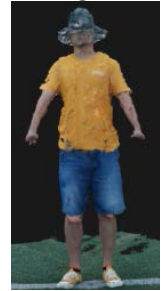

(a)

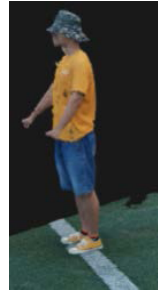

(b)

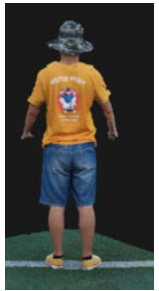

(c)

Fig. 10. Dense clouds.

\subsection{Surface reconstruction}

In this paper, Poisson surface reconstruction is adopted to generate the manikin. The main idea of the algorithm is to reconstruct by using the internal relationship between the directed point set sampled on the model surface and the index function of the model. The key point is that the gradient of the index function is a vector field close to zero (the index function is constant everywhere), so the points around the model surface can be removed. Therefore, Poisson reconstruction is performed by taking the directional point sample as the gradient of the indicator function of the model. The reconstructed front (a), side (b) and back (c) are shown in Figure 11:

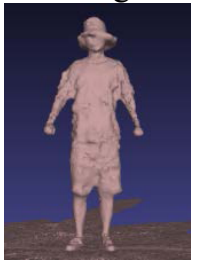

(a)

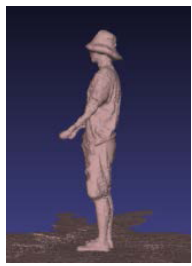

(b)

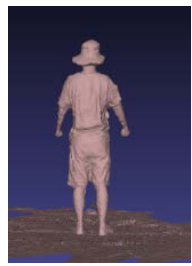

(c)

Fig. 11. Poisson reconstruction rendering. 
From the reconstruction results that the reconstruction of the back is more perfect, the details are complete, the side is the second, the details of the front torso are more perfect, and the upper arms are wrinkled but lack of details.

\section{Conclusion}

This paper describes the pipeline process of 3D reconstruction of mannequin based on two-dimensional image. Among the 50 images in the data set, the chessboard chart is used for camera calibration, the SIFT operator is used for feature extraction and matching, and the incremental motion recovery structure is used to reconstruct the sparse human body point cloud model. On this basis, the depth map of the corresponding image is generated, and the dense point cloud model is formed after the depth map fusion. Poisson surface reconstruction algorithm is selected to griddle the dense point cloud and optimize it to make it relatively smooth. The final experimental results can get the basic human body model, but the details in the upper arm and other parts are lost, which needs to be further improved.

\section{References}

1. Xiao Xue. Human skeleton extraction and 3D reconstruction based on video sequence [D]. Dalian University of Technology, 2009.

2. Izadi S, Kim D, Hilliges $\mathrm{O}$, et al. KinectFusion:real-time $3 \mathrm{D}$ reconstruction and interaction using a moving depth camera[C] .Acm Symposium on User Interface Software \& Technology. 2011.

3. Shi Le. Research on real-time personalized mannequin reconstruction algorithm based on two-dimensional image [D]. Xi'an Engineering University, 2019

4. Wang Li. Research on non-contact anthropometry and modeling technology based on image [D]. Xi'an Engineering University, 2018.2014.

5. Zhang Guangpian, Ji Zhongping. Three-dimensional human body modeling method based on two-dimensional point cloud image [J]. Computer Engineering and applications, 2020556 (19): 205-215

6. Xu Lei. Research on 3D mannequin reconstruction system based on sequence images [D]. Nanjing University of posts and Telecommunications, 2017

7. Yin Hengyu. Research and implementation of image-based 3D modeling algorithm [D]. University of Electronic Science and Technology, 2020.

8. Zhang Zhao. Research on key technologies of 3D reconstruction of mannequin based on SFM [D]. Hubei University of Technology, 2019.

9. Yu Xianchuan,Lv Zhonghua, Hu Dan. Overview of remote sensing image registration technology[J]. Optical precision engineering, ,2013,21(11):2960-2972.

10. Alexander Tscharf. UAV-gestützte Vermessung im Bergbau - Zur Frage der Genauigkeit unter Verwendung von Structure from Motion UAV-based Mine Surveying - On the Question of Accuracy Using Structure from Motion [J]. BHM Berg- und Hüttenmännische Monatshefte,2020,165(W5): 